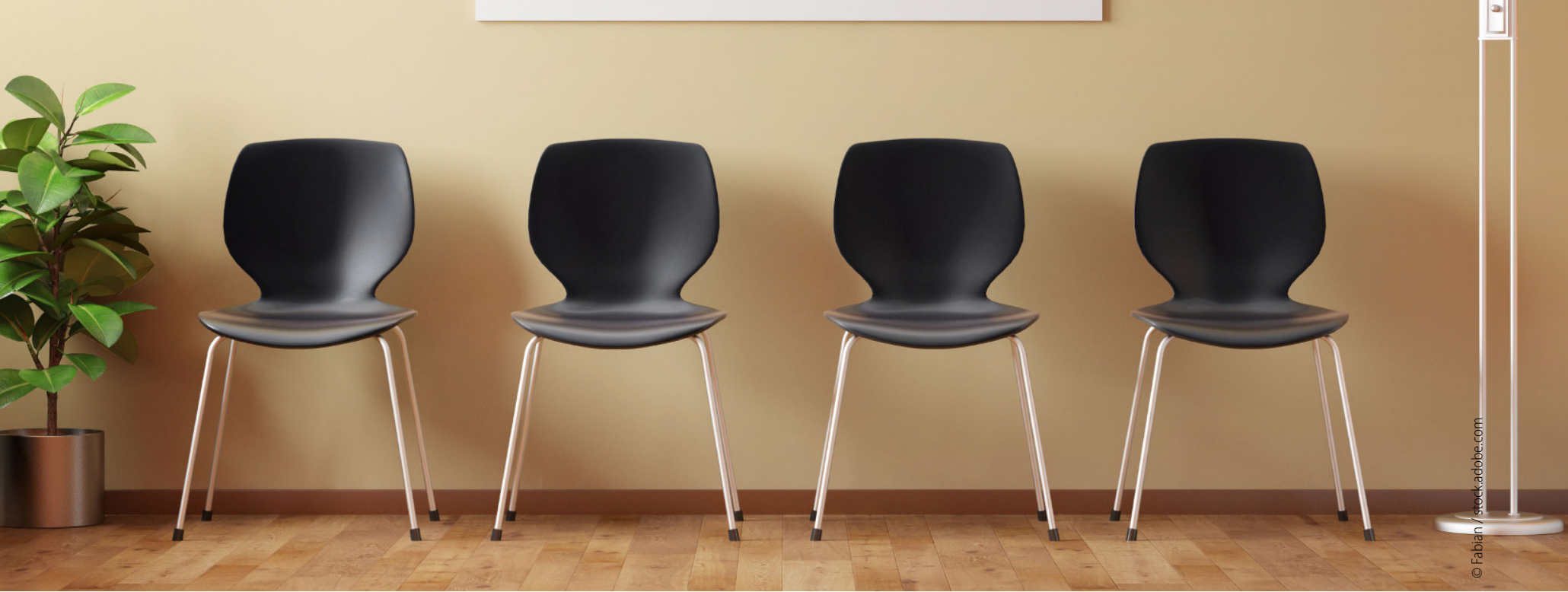

\title{
Entschädigung für Praxisschließung wegen Coronavirus
}

Ein konkretes Tätigkeitsverbot eines Arztes oder Praxismitarbeiters infolge einer SARS-CoV-2-Infektion führt zu einem Erstattungsanspruch gegenüber dem Staat. Alle anderen Ausfallkosten können nur mit Versicherungspolicen abgedeckt werden.

Immer wenn die Gesundheitsbehörde ein konkretes Tätigkeitsverbot gegen einen Mitarbeitenden in einer Arztpraxis ausspricht, der auch namentlich genannt ist, greift zunächst $\$ 56$ Infektionsschutzgesetz. Das heißt, der Arbeitgeber zahlt die Lohnfortzahlung für den Mitarbeitenden mit Tätigkeitsverbot und hat einen Anspruch auf Erstattung durch den Staat, die er an die zuständige Behörde richten muss. Die Kassenärztliche Bundesvereinigung hat für diesen Fall eine Liste der in allen Bundesländern zuständigen Behörden im Internet veröffentlicht (online unter www.kbv.de, Suchbegriff „Praxisschließung bei Coronavirus“ zu finden).

Erstveröffentlichung in URO-NEWS (2020) 24(4):56-57.

best practice onkologie $2020 \cdot 15$ (11): 508-510

https://doi.org/10.1007/s11654-020-00255-y

Online publiziert: 14 . Oktober 2020

(c) Springer Medizin Verlag GmbH, ein Teil von Springer Nature 2020

\section{Anordnung als Voraussetzung}

In Nordrhein-Westfalen erhalten Niedergelassene, die wegen des Coronavirus vorsorglich unter Quarantäne gestellt werden, eine Entschädigung von den Landschaftsverbänden Rheinland (LVR) und Westfalen-Lippe (LWL). Voraussetzung ist, dass die Quarantäne durch die zuständigen Gesundheitsämter angeordnet wurde. LVR und LWL leisten bei Verdienstausfällen von Arbeitnehmern sowie von Selbstständigen inklusive der Freien Berufe. Niedergelassene Ärzte haben Anspruch vom ersten Tag der Quarantäne an und müssen den Antrag auf Entschädigung innerhalb von drei Monaten nach Ende der Quarantäne bei dem für sie zuständigen Landschaftsverband stellen.

\section{Vorleistung des Praxisinhabers}

Bei Praxismitarbeitern und angestellten Ärzten gilt wie bei allen Arbeitnehmern: Die Arbeitgeber müssen als Teil der Entgeltfortzahlung für bis zu sechs Wochen in Vorleistung treten. Sie erhalten die ausgezahlten Beiträge auf Antrag vom Landschaftsverband erstattet. Er zahlt im Bedarfsfall ab der siebten Woche direkt an die Betroffenen. „Die Entschädigung entspricht der Höhe des gesetzlichen Krankengeldes“, teilten LVR und LWL mit. Arbeitnehmer müssen sich nicht selbst um die Entschädigung kümmern, das läuft automatisch über den Antrag des Arbeitgebers.

Was an weiteren Kosten während des Tätigkeitsverbots für einzelne Mitarbeiter noch übrig bleibt, zum Beispiel für Ersatzpersonal, deckt dann die Betriebsschließungsversicherung. 
Dazu gehören auch die Kosten für die Desinfektion sowie für Ermittlungs- und Beobachtungsmaßnahmen.

\section{Was Versicherungen leisten}

Bei dem auf das Gesundheitswesen spezialisierten Versicherungsmakler Ecclesia med gehen derzeit oft Anfragen von niedergelassenen Ärzten ein: Viele erkundigen sich nach Absicherungsmöglichkeiten für den Fall, dass sie die Praxis wegen des Coronavirus schließen müssen - weil sie selbst oder Mitarbeiter sich mit SARS-CoV-2 infiziert haben.

Das Interesse an einer Praxisausfallversicherung sei hoch, berichte Geschäftsführerin Nadja Bürger auf Anfrage von Springer Medizin. „Die Praxisausfallversicherung deckt das Risiko des Praxisinhabers, wenn er die Praxis aufgrund einer behördlichen Anordnung in Zusammenhang mit einem Infektionsfall schließen muss", erläuterte sie.

Bei der Praxisausfallversicherung sei jedoch einiges zu beachten: Meist gebe es bei solchen Policen eine Karenzzeit, sie greifen also nicht vom ersten Tag der Schließung an. Die Deckung greift weiterhin nur, wenn die Praxis aufgrund einer behördlichen Anordnung geschlossen wurde. Anders sieht es aus, wenn der Arzt nur den Verdacht hat, dass er oder einer der Mitarbeiter sich infiziert hat. Selbst wenn ein Mitarbeiter bereits unter Quarantäne steht und die Behörde ein Beschäftigungsverbot ausgesprochen hat, ist das kein Fall für die Praxisausfallversicherung. Denn sie deckt nur die Erkrankung des Arztes selbst.

Es sei aber sinnvoll, auch das Team zu berücksichtigen, betont sie. Schließlich ist es schwierig, eine Praxis zu führen, wenn zwar der Arzt gesund ist, alle anderen jedoch wegen Krankheit ausfallen.

\section{Neue Policen kaum bekannt}

Das Konzept der Praxisausfallversicherung hält Bürger für veraltet. Inzwischen gebe es Betriebsschließungsversicherungen, die den modernen Anforderungen deutlich besser entsprechen. Allerdings seien diese Policen bei niedergelassenen Ärzten noch nicht sehr bekannt - nicht zuletzt deshalb, weil auch viele Versicherungsvermittler sich damit nicht auskennen. Anders sehe es bei Krankenhäusern, Altenheimen oder Kindergärten aus. Sie würden sich schon gegen die Schließung aufgrund von Infektionskrankheiten absichern.

Diese Police greift laut Bürger bereits, wenn der Arzt die Praxis aufgrund einer behördlichen Empfehlung schließt, auch wenn noch keine Anordnung vorliegt. Zudem seien Maßnahmen wie die Desinfektion der Praxis oder eine Teilschließung vom Versicherungsschutz erfasst.

\section{Information an Behörden wichtig}

Egal, ob ein Arzt eine Praxisausfallversicherung oder eine Betriebsschließungspolice hat: Er muss die zuständigen Behörden einschalten und sollte einen vermuteten Infektionsfall möglichst früh seinem Versicherer melden. Wichtig sei die Information, um welchen Erreger es sich handelt und mit welchen Kosten der Arzt durch die Infektion und die Schließung rechne, so Bürger.

Der Praxisinhaber sollte im engen Austausch mit den Behörden bleiben und deren Anweisungen Folge leisten. „Das Schlimmste ist, sich nicht $\mathrm{zu}$ melden, weil man die Konse- quenzen fürchtet“, warnt Bürger. Grob fahrlässiges Verhalten kann den Versicherungsschutz gefährden.

Bei der Praxisausfallversicherung ist der Versicherungsschutz auf die Krankheiten beschränkt, die im Infektionsschutzgesetz aufgelistet sind. Die Betriebsschließungspolice greife dagegen auch bei Infektionen, die noch nicht explizit im Gesetz stehen, so Bürger. „Eine Betriebsschließungspolice muss nicht teuer sein“, erklärt die Expertin. Die Prämie hinge vom Umsatz ab. Schon für einige hundert Euro sei die Deckung zu bekommen.

Ärzte, die sich angesichts des Coronavirus für eine solche Police interessieren, sollten nicht allzu lange warten, empfiehlt Bürger. Denn manche Anbieter haben den Versicherungsschutz für Neuabschlüsse in Bezug auf Corona bereits eingeschränkt.

Versicherer geben sich bei dem Thema Praxisausfallversicherung oder Betriebsschließungsversicherung häufig zugeknöpft. Pauschale Aussagen könne man zu dem Thema nicht machen, da es sich um individuelle Vereinbarungen handle und die Gründe für eine Schließung sehr unterschiedlich gelagert sein könnten, teilt die Axa auf Anfrage mit. „Wir empfehlen unseren Kunden, sich für ihre individuellen Fragen vorab mit ihrem Vertriebspartner in Verbindung zu setzen." Damit meint die Axa Versicherungsvertreter oder Makler.

Ilse Schlingensiepen 
Hier steht eine Anzeige.

Springer 